\title{
On Hierarchical Propositions
}

\section{Giorgio Sbardolini ${ }^{1}$}

Received: 7 October 2017 / Accepted: 1 April 2019/ Published online: 26 April 2019

(C) The Author(s) 2019

\section{Abstract}

There is an apparent dilemma for hierarchical accounts of propositions, raised by Bruno Whittle (Journal of Philosophical Logic, 46, 215-231, 2017): either such accounts do not offer adequate treatment of connectives and quantifiers, or they eviscerate the logic. I discuss what a plausible hierarchical conception of propositions might amount to, and show that on that conception, Whittle's dilemma is not compelling. Thus, there are good reasons why proponents of hierarchical accounts of propositions (such as Russell, Church, or Kaplan) did not see the difficulty Whittle raises.

Keywords Paradoxes · Propositions · Type theory · Simple and ramified hierarchy

\section{Intensional Paradoxes}

There are several paradoxes concerning propositions. ${ }^{1}$ Following Whittle [15], let us focus on two. The first is what we may call Whittle's paradox:

suppose that $R$ is a property that applies to propositions of the form $F(a)$ (for some property $F$ and object $a$ ) iff $F$ does not itself apply to the proposition in question. We then consider a proposition $R(b)$ (for some object $b$ ) and ask: does $R$ apply to this proposition? Suppose first that it does. Then, by the definition of $R$, it must not apply to it. So $R$ does not apply to this proposition. But thenby the definition of $R$ again $-R$ must apply to the proposition—contradiction! (Whittle [15, p. 217])

The second is an intensional version of the Liar paradox. Consider the proposition expressed by the sentence ' $\forall p(A p \rightarrow \neg T p)$ ', which states that for any proposition $p$, if $p$ is asserted by Epimenides, then $p$ is not true. Suppose furthermore that

\footnotetext{
${ }^{1}$ The most famous include Russell [12, 13], Myhill [8], Prior [9], Kaplan [7].

Giorgio Sbardolini

g.sbardolini@uva.nl

1 ILLC, University of Amsterdam, Science Park 107, 1098 XG Amsterdam, The Netherlands
} 
Epimenides asserts only the proposition expressed by that sentence. Is this proposition true or not? If it is, then, since it is asserted by Epimenides, it is not true. If it is not true, then since it is the only proposition asserted by Epimenides, it is true. Hence the proposition expressed by ' $\forall p(A p \rightarrow \neg T p)$ ' seems to be both true and not true.

We shall follow Whittle and assume that propositions are structured entities. This gives some indication of what Whittle means - in the passage above-when he says that propositions "have a form". However, it should be emphasized that paradoxes concerning propositions arise whether or not propositions are structured, and depend only on logical features of the notions of a proposition and of propositional quantification (as we shall see below). Having made this point, for the rest of the paper I shall be articulating a way to understand the idea that propositions live in a hierarchy, which is a popular strategy against paradoxes like the ones above.

Whittle raises a dilemma for (some) hierarchical approaches, which I'll discuss below. I believe, however, that the dilemma does not point to a general difficulty for hierarchical accounts of propositions. Instead, it appears to arise from a conflation of two assumptions: (i) the idea that complex objects, like propositions, are built out of simpler objects, and (ii) the philosophical conception motivating some 'ramifiers', like Russell [13], Church [1], and Kaplan [7], which is presumably centered around something like a Vicious Circle Principle. Although these ideas might be connected, it is the ban against circularity that justifies the hierarchy, not the claim that propositions are structured. So there's no reason to think that the dilemma Whittle raises is compelling for the ramifiers. In the conclusion I shall raise some independent worries concerning Whittle's own hierarchical account of propositions.

\section{The Dilemma}

Why go hierarchical? Intuitively, because requiring that in one way or the other propositions are arranged hierarchically helps us avoid a (vicious) circularity in paradoxical reasoning. ${ }^{2}$ In Whittle's paradox, circularity is hidden in the definition of $R$ : a property that applies to a proposition $F(a)$ iff $F$ does not apply to $F(a)$. In the intensional version of the Liar, more subtly, we assume that ' $\forall p(A p \rightarrow \neg T p)$ ' expresses a proposition, and that this proposition is in the range of the universal quantifier that is used to express it. Reasoning from these definitions dramatically brings out the circularity: the property thus defined applies to some propositions if and only if it doesn't, and the proposition thus expressed is true if and only if it isn't.

Famously, the most general ban against circularity is Russell's Vicious Circle Principle, which in the intentions of Russell offers philosophical justification for the Ramified hierarchy. The VCP is notoriously controversial, and its precise formulation is unclear. ${ }^{3}$ But weaker claims than the VCP justify less cumbersome theories

\footnotetext{
${ }^{2}$ It may not be that all paradoxes involve some circularity, but Russell certainly thought they all did. Let us set aside the question whether hierarchical accounts succeed in dealing with any paradoxes, and focus on what motivates them.

${ }^{3}$ See among others, Gödel [3], Quine [10], Goldfarb [4], Jung [6], Hodes [5].
} 
than the Ramified hierarchy, and these simpler theories sometimes enjoy wide acceptance. For instance, requiring that sets are arranged hierarchically helps us avoid a circularity hidden in the definition of $r$, a set whose members are any sets $x$ such that $x$ is not a member of $x$. The ban in ZFC against self-membered sets can be seen as a weak version of Russell's all-out ban against circularity, one that doesn't rely on the VCP, arises naturally from a well-motivated conception of sets, and provides justification for Zermelo's widely accepted Cumulative hierarchy.

Conceptually, type theories and set theories are very different. A set theory is a description of the universe of sets, and sets are distinctive mathematical objects. Set theories are used in mathematics to address several questions of existence and consistency. Type theories have been devised to regiment certain formal features of languages (with a recursively specified syntax). They are used to categorize linguistic expressions according to their logical (i.e. combinatorial) features. Still, there is some continuity between sets and types. In an extensional semantics, the types of wellformed expressions correspond to certain kinds of sets in a precise way. To say that a name $\eta$ is type $e$ is to say that there is a set $E$ of individuals and that the extension of $\eta$ (its referent) is an element of $E$. To say that a generalized quantifier $\theta$ is type $\langle\langle e, t\rangle,\langle\langle e, t\rangle, t\rangle\rangle$ is to say that the extension of $\theta$ is a relation (i.e. still a set) $T$ between two sets of individuals $A$ and $A^{\prime}$ (for example, if $\theta$ is the universal quantifier, $T$ is the subset relation, thus asserting that all members of $A$ are members of $A^{\prime}$ ). Finally, to say that a predicate $\phi$ is type $\langle e, t\rangle$ is to say that there is a set $F$ of sets of individuals and that the extension of $\phi$ is an element of $F$. And so on. All these claims of existence have to be licensed, of course, by a coherent conception of sets, which is typically the one offered by Zermelo's foundations.

So, there is one general reason to go hierarchical, and this is to avoid some kind of circular reasoning that is present in the paradoxes-at least according to some authors. But then there are very different hierarchies, and very different conceptions of the hierarchically arranged objects (whether they are sets or propositions). Often, especially in work on the set-theoretic paradoxes, 'circular' reasoning is discussed under the heading "impredicative reasoning". There is widespread agreement that impredicative reasoning in mathematics is largely acceptable up to minimal constraints — such as those implemented by Zermelo's axioms (i.e. Separation in particular). It is much less common to think that impredicative reasoning is unacceptable across the board, as the VCP would seem to require. Just how much circularity (impredicativity) is acceptable may vary depending on the subject matter, and is a subtle point on which different philosophical conceptions, motivating different hierarchical accounts, can be brought to bear.

As we shall see below in more details, the Ramified hierarchy is a subsystem of the Simple hierarchy: one in which certain order-theoretic restrictions are imposed. The Simple hierarchy itself is a subsystem of Zermelo's hierarchy: one in which certain other restrictions are imposed, as pointed out long ago by Gödel [3]. Let's suppose that Zermelo's hierarchy is the upper end of hierarchical systems-i.e. the most liberal we are willing to consider (within the realm of consistency). Hierarchical accounts of any kind of objects (propositions or sets) correspond to a more or less restrictive subsystem of Zermelo's hierarchy. Each of these accounts will have to 
find its conceptual motivation in the underlying metaphysics of the hierarchically arranged objects.

The particular hierarchy discussed by Whittle appears to be sort of intermediate between Ramified and Simple:

At level 0, one would start with objects that are neither properties nor propositions. At level 1, one would have properties that apply to these and propositions that are about them. At level 2, one would have properties that apply to the things that are levels 0 and 1, and propositions that are about them-and so on. (Whittle [15, p. 217])

Visually, we get the following picture, where I indicate the levels, the corresponding linguistic expressions as they appear in Whittle's paradox, and their type:

$\begin{array}{lll}\text { Level } 0 & a, \ldots & \text { basic type } \\ \text { Level 1 } & F, \ldots & \text { type of properties of things of level } 0 \\ \text { Level 1 } & F(a), \ldots & \text { type of propositions about things of level } 0 \\ \text { Level 2 } & R, \ldots & \text { type of properties of things of level } 0 \text { or } 1\end{array}$

The sketch continues with another line labelled 'level 2', for expressions with the type of propositions about things of level 0 or 1 , and so on. Whittle points out, correctly, that this hierarchy helps us against Whittle's paradox. However, he raises the following dilemma about it:

On a hierarchical account, no property can apply to a proposition that is itself built out of that property. But what then about propositions of the form $\neg \neg p$, for example? For here we have an operator-negation-applying to a proposition that is itself built out of that operator. But if, across the board, propositions of the form $F(F(a))$ are disallowed, then surely propositions of the form $\neg \neg p$ should be too. For why should negation be allowed to simply exempt itself from the hierarchical restrictions? ... On the other hand, applying these to negation would seem to eviscerate logic. For it is surely an important and central feature of our concept of negation that it can be iterated. ... We thus have a dilemma: either render the approach ad hoc (by simply exempting certain connectives and quantifiers from the hierarchical restrictions) or eviscerate logic (by not doing that!). Needless to say, neither option would seem to be very attractive. (Whittle [15, pp. 219-220])

The dilemma, if compelling, is a threat to hierarchical accounts of propositions: Whittle mentions Russell [13] and Church [1] as two theories for which this dilemma would arise. But I shall argue that the dilemma is not compelling, and indeed that there's a good reason why it does not arise for Russell or Church. The dilemma depends on the specific conception of the nature of propositions that Whittle adopts, and that justifies the hierarchy we sketched above. However, such conception is arguably not in the background of Russell and Church, nor of other famous ramifiers like Kaplan [7], and it does not motivate their theories-nor does Whittle claim that it would. As Whittle offers no independent argument for the particular conception he puts forward, there's no reason to think that ramifiers are affected by the dilemma. 


\section{Hierarchies}

Whittle's paradox is in effect a property version of Russell's paradox: the role played by propositions in the argument is inessential. To see this, consider the property version of Russell's paradox. First, assume a Naïve Comprehension Principle NC1:

$$
\exists P \forall Q(P(Q) \leftrightarrow \phi(Q))
$$

where $Q$ ranges over properties. The property version of Russell's paradox follows from consideration of the property $R$ that applies to all and only those properties that don't apply to themselves. An instance of NC1 straightforwardly entails that:

$$
\forall Q(R(Q) \leftrightarrow \neg Q(Q))
$$

i.e. a property $R$ applies to all and only those properties $Q$ that don't apply to themselves. Contradiction follows immediately by instantiating the quantified variable on $R$ itself.

Regarding Whittle's paradox, assume a Naïve Comprehension Principle NC2, which differs (inessentially) from $\mathrm{NC} 1$ in that the universally quantified variable is now interpreted as ranging over propositions:

$$
\exists P \forall q(P(q) \leftrightarrow \phi(q))
$$

On a different instance of $\phi, \mathrm{NC} 2$ gives:

$$
\forall q(R(q) \leftrightarrow \exists F \exists a(q=F(a) \wedge \neg F(q))
$$

i.e. a property $R$ applies to all and only those propositions $q$ such that for some property $F$ and object $a, q$ is the proposition that $F(a)$ and $F$ does not apply to $q$. Contradiction follows in the way we already said.

In a sense, these are different versions of Russell's paradox: whether the universally quantified variable ranges over sets, properties, or propositions, it makes little difference. As is well known, Russell's paradox does not arise in Zermelo's hierarchy, because Naïve Comprehension is not licensed by Zermelo's axioms and the existential claims displayed on the previous paragraph are shown to be false. A fortiori, the paradox does not arise in the Simple and Ramified hierarchies (which are stricter than Zermelo's).

The philosophical justification for the Simple hierarchy can be put as follows: it is very natural to distinguish between predicates that apply to basic things (is a natural number), predicates that apply to sets of basic things (is equinumerous with the set of natural numbers), predicates that apply to sets of sets of basic things (is equinumerous with the set of subsets of natural numbers), etc. It is just as natural to distinguish between predicates of basic things, predicates of properties of them, predicates of properties of properties of them, etc. Simple type theory imposes these distinctions on logical languages: there are level 0 predicates (of basic things), level 1 predicates (of sets/properties of them), level 2 predicates (of sets/properties of sets/properties of them), etc. Violation of these distinctions leads either to nonsense or to necessary falsehood (e.g. outright contradiction, or inconsistency with Cantor's theorem). Since predicates can be divided in this way, classes of things to which they may apply can 
be divided accordingly. Thus we have a basic class of things to which level 0 predicates apply, a class of things to which level 1 predicates apply, and so on. Things divided into classes in this way are said to form a Simple hierarchy. It applies to sets, and to properties. Accordingly, I will be talking about levels of predicates of hierarchically arranged things, as well as levels of hierarchically arranged things.

Recall that in Whittle's hierarchy, propositions about level $n$ stuff live at level $n+1$. This might be motivated by the idea that propositions are built out of properties (plus some other things). Properties are directly tied to predication, for predicates express properties and have sets as extensions, and since predicates are typed, properties are arranged hierarchically. There's a broader philosophical question about the relation between predication and propositions - the objects of thought. A popular view is that properties are the middleman in this relation: they are expressed by predicates, but they are also parts of propositions. On this view, presumably propositions themselves will be arranged hierarchically: one does not run into a proposition that contains a property of level $n$ unless one is at least at level $n$. However, this is one view among many, and the nature of the relation between predication and propositions remains controversial. The mereological model I just sketched of the relation between propositions and properties is by no means common ground. If properties are not parts of propositions, we have as yet no apparent reason to think that propositions are arranged hierarchically.

Whittle's paradox by itself gives no reason to think that propositions are arranged hierarchically: formally, they could all live at any level of the Simple hierarchy (e.g. the lowest): paradox is avoided so long as we distinguish between properties of propositions (like $F$ ) and properties of properties of propositions (like $R$ ) -as I remarked above, quantification over propositions in Whittle's paradoxical argument is not essential, as it is a property version of Russell's paradox.

There are reasons to think that propositions are arranged hierarchically thoughbut in a different sense of 'hierarchy'. So far, we haven't touched on Russell's Ramified hierarchy. This theory is helpful if one is to account for other kinds of paradoxes, i.e. those that, as Russell knew well, might arise even within the restrictions of Simple type theory. The Intensional version of the Liar paradox, given above, is one such example. It is routine to check that the expression ' $\forall p(A p \rightarrow \neg T p)$ ' is syntactically well-formed, by the lights of the Simple theory of types. Intuitively, there are no self-applying properties nor self-membered sets, which is what we were interested in avoiding, and yet the Intensional Liar paradox still follows. To handle this case we need ramification, at least according to Russell, Church, and Kaplan. ${ }^{4}$

What's the point of ramification? That's controversial, and it goes by different interpretations of the Vicious Circle Principle. But the general idea is that certain objects, like the proposition expressed by ' $\forall p(A p \rightarrow \neg T p)$ ' are defined in a way that "presupposes" the collections of all objects to which it belongs —in this case, we define a proposition as the one expressed by a sentence that contains a quantifier

\footnotetext{
${ }^{4}$ However, the more popular view nowadays is that we don't need ramification at all. It was Ramsey [11] who suggested that these paradoxes don't have a solution in pure logic (or type theory). According to Ramsey, contradiction follows in these cases from some faulty assumption perhaps about the notions of truth and predication.
} 
over all propositions (including the definiendum itself). According to the ramifiers, there is something bad about this definition, although it has proven hard to say what exactly. But the point that matters here is that whereas the Simple hierarchy is motivated by the idea that classes of things are "formed" in stages (to use the popular metaphor for Zermelo's hierarchy), the Ramified hierarchy is motivated by a philosophical conception of quantification, and of how certain objects are "defined" by means of the quantifiers. ${ }^{5}$

As before, it is helpful to think of hierarchies starting from thinking about predicates. Consider predicates of propositions such as is true or is asserted by Epimenides. Given the underlying conception of the Simple hierarchy I sketched, we may assume that all predicates of propositions have the same level. The class of things to which they apply is the class of propositions-there is one such class from the perspective of the Simple hierarchy. Both is true and is asserted by Epimenides express properties of propositions and they both have sets of propositions (subsets of the class of propositions) as extensions. If so, then it's plausible to think that slightly more complex predicates of propositions have the same level too. For instance, predicates like is true and asserted by Epimenides, and is such that if it is asserted by Epimenides then it's not true.

Ramification comes in next. Along with the distinction of predicates into levels, we are going to distinguish each level into different orders. For an example (coming from Russell [14]; see also Giaquinto [2]), compare these two predicates:

i. $\quad$ is a good military strategist.

ii. has all the properties of a great general.

We may suppose that Napoleon was a good strategist, and that he had all the properties of a great general. The point to notice is that (ii) expresses a property that Napoleon had only if he had the property expressed by (i). The opposite direction doesn't hold, for one may well be a good strategist without having all the properties of a great general. Predicates (i) and (ii) are of the same level since they both apply to Napoleon. But we will say they are of different orders.

In particular, let's say that if is true, and is asserted by Epimenides, are order $\alpha$, then so are the predicates is true and asserted by Epimenides, and is such that if it is asserted by Epimenides then it's not true. For a proposition is described by one of these predicates just in case it belongs to some subset or other of the class of all propositions. However, the predicates is true iff everything is false, is true iff nothing asserted by Epimenides is true, etc., are level $\alpha+1$. Intuitively, a proposition is described by one of these second kinds of predicates just in case some condition holds of the class of all propositions "as a whole". One might see each predicate as being implicitly "context-sensitive", so that is true really should read: is of order $\alpha$ and true. Then a predicate such as is true iff every proposition of order $\alpha$ is false is implicitly of order $\alpha+1$, whereas is true iff every proposition of order $\alpha+1$ is false is implicitly of order $\alpha+2$. And so on.

\footnotetext{
${ }^{5}$ As I mentioned, there is controversy surrounding what exactly the VCP says and why should it be true. But it is not controversial that the VCP is a ban against "definitions" of something $x$ that "presuppose" the class of things which $x$ belongs to: Russell was at least that much clear.
} 
Notice that all these higher order predicates are still predicates of propositions, so they are all the same level. Yet, intuitively, they all "presuppose" sets of propositions of increasing orders. In other words, from the perspective of the Simple hierarchy, all propositions form a single class, i.e. all propositions live at the same level. But now, within that level, we distinguish orders of predicates of propositions. Hence propositions do not form a single class from the perspective of the Ramified hierarchy. There will be a basic class of propositions to which order 0 predicates apply, a class of propositions to which order 1 predicates apply, and so on. A hierarchy that combines the division into levels and into orders is called a Ramified hierarchy. In the Ramified hierarchy of propositions, the Intensional Liar paradox does not arise. For suppose that the predicate is such that if it is asserted by Epimenides then it's not true is order $\alpha$. Suppose that $q$ is the proposition expressed by ' $\forall p(A p \rightarrow \neg T p)$ ', namely that $q$ is described by a predicate of order (at least) $\alpha+1$ : the predicate is true iff all propositions asserted by Epimenides are not true. It follows that $q$ belongs to the class of propositions which predicates of order $\alpha+1$ apply to. Then a predicate of order $\alpha$ is such that if it is asserted by Epimenides then it's not true does not apply to $q$, for $q$ does not belong to the class of propositions which predicates of order $\alpha$ apply to.

The Simple hierarchy arises from consideration of the difference between predicating something of a thing, predicating something of a class of things, predicating something of a class of classes of things, and so on. Violation of restrictions imposed by the Simple hierarchy may lead to inconsistency, as we said. The Ramified hierarchy, in the way I presented it, arises from the consideration that certain acts of predication are, in some sense, quantificational: they presuppose a class of things. Violation of restrictions imposed by the Ramified hierarchy may lead to the intensional paradoxes. Of course there are many details I didn't discuss, and other conceptions of these two hierarchies are possible, but I have offered a plausible sketch of the philosophical considerations motivating them. I contend that the sketch I offered is, albeit vague, somewhat faithful to Russell's original intentions-see Goldfarb [4] and Hodes [5] for interpretations of Russell according to which ramification is motivated by the theory of higher order quantification.

Hierarchies (of levels or of orders) are helpfully understood as ways of classifying predicates based on their logical features, and classes of things which these predicates are applied to are classified accordingly. Hierarchies do not (necessarily) arise from putting together complex objects out of simpler objects. I have presented conceptions of the Simple and the Ramified hierarchy on which neither is motivated by mereological composition. As we shall see, this undermines Whittle's dilemma. ${ }^{6}$

\footnotetext{
${ }^{6}$ To be sure, the conception of the Ramified hierarchy I sketched is underdescribed, and one should well wonder why propositions depend on syntactical features of their definitions, in the way the VCP seems to suppose. If propositions are structured objects, one might see why. But the assumption I have made to justify the Ramified hierarchy is one of metaphysical dependence, not one about the intrinsic nature of propositions. Hence, metaphysical accounts of propositions other than the structured account seem prima facie possible, which would support the Ramified hierarchy.
} 


\section{Dilemma Revisited}

Whittle's dilemma arises from a difficulty that potentially targets any logical operator, but negation in particular. Since the proposition that $\neg p$ is built out of negation and $p$, then intuitively $\neg p$ is going to live upstairs, and $p$ downstairs. Accordingly, the proposition that $\neg \neg p$ lives twice removed from $p$. That's a problem because the inner occurrence of ' $\neg$ ', since it applies to an object living downstairs, has to denote a different operator than the outer occurrence of ' $\neg$ ', since the latter applies to an object living upstairs. And so on. In effect, negation cannot be iterated-which, as Whittle points out, is tantamount to say that we don't really have a negation.

Now, why should negation push one up in the hierarchy? The thought that increasing grammatical complexity forces one up the hierarchy is compelling, perhaps, if one goes up the stairs by way of composing propositions out of parts. However, the conceptions I sketched for the Simple and the Ramified hierarchies are not motivated by this thought.

Suppose we want to make sense of the idea that $\neg p$ lives upstairs, and $p$ downstairs. Suppose that the floors in this metaphor are levels. We would be saying that $\neg p$ is level $n$, and $p$ is level $n-1$. But that's not justified: in the Simple hierarchy of propositions, levels go up by consideration of sets (or properties) of things of a certain level. So we have propositions at one level, and sets or properties of propositions at the next level, and so on. Negation does not take from a thing to a set of things, and neither do conjunction, disjunction, etc. Thus, there's a principled reason why the logical connectives are exempted from the restrictions of the Simple hierarchy.

Suppose instead that the floors in the metaphor are orders. We would be saying that $\neg p$ is order $\beta$, and $p$ is order $\beta-1$. But that's not justified either, at least not on the quantificational reading of the VCP sketched above. In the Ramified hierarchy of propositions, orders go up by consideration of classes of propositions which we may presuppose in order to define more and more propositions. So we have propositions of an order, and propositions defined by presupposing the class of all propositions of that order at the next order, and so on. There's no sense in which negation "presupposes" classes of propositions, and neither do conjunction, disjunction, etc. It is only quantifiers that trigger presuppositions of this sort, because they seemingly require a domain of propositions for the variables to range over. So, even by the lights of the Ramified hierarchy, there's a principled reason why increasing grammatical complexity, at least by means of the connectives, does not push one up the hierarchy.

In effect, absent an independent argument why the Simple or the Ramified hierarchy should be interpreted according to considerations of mereological complexity, there's no reason to assume that negation and the other connectives interact at all with either hierarchy. Whittle complains (p. 220) that Russell, and commentators such as Gödel and Church, didn't recognize that the exemption of the connectives from hierarchical restrictions is ad hoc, and didn't notice the dilemma he raised. But I made the case that Whittle's difficulty arises only for a specific conception of the nature of propositions, that apparently motivates the particular hierarchy Whittle is working with, on which the relation between levels is understood as partly a mereological matter. To be sure, whether Russell assumed (at various points in his career) that 
propositions have parts, and whether this view had some role in preparing his ramified approach to the antinomies, are matters for discussion. In any case, there's good reason why the logical operators are exempt from either hierarchy, why Russell's theory is not ad hoc (at least, not in the respects I addressed here), and why Gödel and Church didn't notice Whittle's dilemma (although they did notice other difficulties for Russell's theory).

\section{Conclusion}

Whittle's difficulty concerning negation is real, if one adopts the particular conception Whittle is working with, but no compelling reason to do so is offered. To solve the difficulty, Whittle sketches a positive proposal in the second part of the paper, about which I shall now make a couple of brief comments.

Whittle's suggestion is to build propositions out of functions, rather than properties (so, we are still under the assumption that propositions are structured objects): "rather than building the proposition that John is tall, for example, out of John, together with the property of being tall, we should instead build it out of John together with the 'tallness function', i.e. the function that sends tall things to the truth value $t$, and everything else to the truth value $f$ " (pp. 220-221). Insofar as functions correspond to their characteristic sets, the Simple hierarchy applies to functions for the same reasons it applies to the extensions of predicates, and so Whittle is recommending a Simple hierarchy. As such, I doubt that it leads to a solution to all intensional paradoxes. In effect, Whittle does not discuss how his proposal is meant to address the Intensional Liar paradox.

There is another, more basic problem. Functions are extensionally individuated, and propositions are not. Suppose, following the familiar example, that all and only animals with hearts are animals with kidneys. Then the function $h$ that sends all and only animals with a heart to $t$ is identical to the function $k$ that sends all and only animals with kidneys to $t$, because $h$ and $t$ have the same extension. Hence $h$ and $t$ are the same function. Thus the mereological complex built out of the function $h$ and Felix the cat (plus whatever else is needed), is the same mereological complex built out of the function $k$ and Felix the cat (plus, etc.). But the proposition that Felix is an animal with a heart is distinct from the proposition that Felix is an animal with kidneys. Perhaps one should think of "functions" in Whittle's sense as intensionally individuated, i.e. something closer to propositional functions in Russell's sense. But Whittle explicitly rejects this move (see his footnote 12 ). So it's unclear how the proposal should lead to a plausible theory of propositions.

The case for Whittle's proposal, in any case, is undermined by the main point of this paper. It is only by adopting a very peculiar conception of logical hierarchies, and one for which no motivation is in the offing, that any difficulties arise of the sort that lead to Whittle's dilemma. I offered plausible (if not well-motivated) conceptions of the Simple and Ramified hierarchies, arguably somewhat close to what the original ramifiers had in mind, and neither leads to the difficulty raised by Whittle. 
Funding Information This work has received funding from the European Research Council (ERC) under the European Union's Horizon 2020 research and innovation programme (grant agreement No 758540) within the project From the Expression of Disagreement to New Foundations for Expressivist Semantics.

Open Access This article is distributed under the terms of the Creative Commons Attribution 4.0 International License (http://creativecommons.org/licenses/by/4.0/), which permits unrestricted use, distribution, and reproduction in any medium, provided you give appropriate credit to the original author(s) and the source, provide a link to the Creative Commons license, and indicate if changes were made.

\section{References}

1. Church, A. (1976). Comparison of Russell's Resolution of the semantical Antinomies with that of Tarski. Journal of Symbolic Logic, 41(4), 747-760.

2. Giaquinto, M. (2002). The search for certainty. Oxford: Clarendon Press.

3. Gödel, K. (1944). Russell's mathematical logic. In Schlipp, P.A. (Ed.) The philosophy of Bertrand Russell (pp. 125-153). New York: Tudor Publishing Company.

4. Goldfarb, W. (1989). Russell's reasons for ramification. In Savage, C.W., \& Anderson, C.A. (Eds.) Rereading Russell: essays on Bertrand Russell's metaphysics and epistemology (pp. 24-40). Minneapolis: University of Minnesota Press.

5. Hodes, H. (2015). Why Ramify? Notre Dame Journal of Formal Logic, 56(2), 379-415.

6. Jung, D. (1999). Russell, presupposition, and the vicious circle principle. Notre Dame Journal of Formal Logic, 40(1), 55-80.

7. Kaplan, D. (1995). A problem in possible world semantics. In Raffman, D., Sinnott-Armstrong, W., Asher, N. (Eds.) Modality, morality and belief: essays in honor of Ruth Barcan Marcus (pp. 41-52). Cambridge: Cambridge University Press.

8. Myhill, J. (1958). Problems arising in the formalization of intensional logic. Logique et Analyse, 1, 78-83.

9. Prior, A. (1961). On a family of paradoxes. Notre Dame Journal of Formal Logic, 2, 16-32.

10. Quine, W.V.O. (1969). Set Theory and Its Logic. Cambridge: Belknap Press.

11. Ramsey, F.P. (1926). The foundations of mathematics. Proceedings of the London Mathematical Society, 2(25), 338-384. Reprinted in F. P. Ramsey, Foundations, London: Routledge and Kegan Paul, $152212,1978$.

12. Russell, B. (1903). The Principles of Mathematics. Cambridge: Cambridge University Press.

13. Russell, B. (1908). Mathematical logic as based on a theory of types. American Journal of Mathematics, 30, 222-262.

14. Russell, B. (1971). Introduction to mathematical philosophy. Simon and Schuster: New York.

15. Whittle, B. (2017). Hierarchical propositions. Journal of Philosophical Logic, 46, 215-231.

Publisher's Note Springer Nature remains neutral with regard to jurisdictional claims in published maps and institutional affiliations. 